\title{
Some inequalities for a LNQD sequence with applications
}

\author{
Yongming $\mathrm{Li}^{i^{*}}$, Jianhua Guo' and Naiyi Li
}

${ }^{*}$ Correspondence: lym1019@163.com

${ }^{1}$ Department of Mathematics, Shangrao Normal University,

Shangrao, 334001, China Full list of author information is available at the end of the article

\begin{abstract}
In this paper, some inequalities for a linearly negative quadrant dependent (LNQD) sequence are obtained. As their application, the asymptotic normality of the weight function estimate for a regression function is established, which extends the results of Roussas et al. (J. Multivar. Anal. 40:162-291, 1992) and Yang (Acta. Math. Sin. Engl. Ser. 23(6):1013-1024, 2007) for the strong mixing case to the LNQD case.
\end{abstract}

MSC: $60 \mathrm{E} 15 ; 62 \mathrm{G} 08 ; 62 \mathrm{E} 20$

Keywords: LNQD sequence; characteristic function inequality; exponential inequality; moment inequality; asymptotic normality

\section{Introduction}

We first recall the definitions of some dependent sequences.

Definition 1.1 (Lehmann [1]) Two random variables $X$ and $Y$ are said to be negative quadrant dependent (NQD) if

$$
P(X \leq x, Y \leq y) \leq P(X \leq x) P(Y \leq y) \quad \text { for any } x, y \in R .
$$

A sequence of random variables $\left\{X_{n}, n \geq 1\right\}$ is said to be pairwise negatively quadrant dependent (PNQD) if every pair of random variables in the sequence is NQD.

Definition 1.2 (Newman [2]) A sequence $\left\{X_{n}, n \geq 1\right\}$ of random variables is said to be linearly negative quadrant dependent (LNQD) if for any disjoint subsets $A, B \subset Z^{+}$and positive $r_{j}^{\prime}$ 's, $\sum_{k \in A} r_{k} X_{k}$ and $\sum_{j \in B} r_{j} X_{j}$ are NQD.

Definition 1.3 (Joag-Dev and Proschan [3]) Random variables $X_{1}, X_{2}, \ldots, X_{n}$ are said to be negatively associated (NA) if for every pair of disjoint subsets $A_{1}$ and $A_{2}$ of $\{1,2, \ldots, n\}$,

$$
\operatorname{Cov}\left(f_{1}\left(X_{i} ; i \in A_{1}\right), f_{2}\left(X_{j} ; j \in A_{2}\right)\right) \leq 0,
$$

where $f_{1}$ and $f_{2}$ are increasing for every variable (or decreasing for every variable) so that this covariance exists. An infinite sequence of random variables $\left\{X_{n} ; n \geq 1\right\}$ is said to be NA if every finite subfamily is NA.

\section{Springer}

(0) 2012 Li et al.; licensee Springer. This is an Open Access article distributed under the terms of the Creative Commons Attribution License (http://creativecommons.org/licenses/by/2.0), which permits unrestricted use, distribution, and reproduction in any medium, provided the original work is properly cited. 
Remark 1.1 (i) If $\left\{X_{n}, n \geq 1\right\}$ is a sequence of LNQD random variables, then $\left\{a X_{n}+b, n \geq\right.$ $1\}$ is still a sequence of LNQD random variables, where $a$ and $b$ are real numbers. (ii) NA implies LNQD from the definitions, but LNQD does not imply NA.

Because of wide applications of LNQD random variables, the concept of LNQD random variables has received more and more attention recently. For example, Newman [2] established the central limit theorem for a strictly stationary LNQD process; Wang and Zhang [4] provided uniform rates of convergence in the central limit theorem for LNQD sequence; Ko et al. [5] obtained the Hoeffding-type inequality for LNQD sequence; Ko et al. [6] studied the strong convergence for weighted sums of LNQD arrays; Wang et al. [7] obtained some exponential inequalities for a linearly negative quadrant dependent sequence; Wu and Guan [8] obtained the mean convergence theorems for weighted sums of dependent random variables. In addition, from Remark 1.1, it is shown that LNQD is much weaker than NA and independent random variables. So, it is interesting to study some inequalities and their applications to a regression function for LNQD sequence.

The main results of this paper depend on the following lemmas.

Lemma 1.1 (Lehmann [1]) Let random variables $X$ and $Y$ be NQD, then

(i) $\mathrm{E} X Y \leq \mathrm{E} X \mathrm{E} Y$;

(ii) Iff and $g$ are both nondecreasing (or both nonincreasing) functions, then $f(X)$ and $g(Y)$ are NQD.

Lemma 1.2 (Zhang [4]) Suppose that $\left\{X_{n} ; n \geq 1\right\}$ is a sequence of LNQD random variables with $\mathrm{E} X_{n}=0$. Then for any $p>1$, there exists a positive constant $D$ such that

$$
\mathrm{E}\left|\sum_{i=1}^{n} X_{i}\right|^{p} \leq D \mathrm{E}\left(\sum_{i=1}^{n} X_{i}^{2}\right)^{p / 2}
$$

\section{Main results}

Now, we state our main results with their proofs.

Theorem 2.1 Let $X$ and $Y$ be NQD random variables with finite second moments. Iff and $g$ are complex-valued functions defined on $R$ with bounded derivatives $f^{\prime}$ and $g^{\prime}$, then

$$
|\operatorname{Cov}(f(X), g(Y))| \leq\left\|f^{\prime}\right\|_{\infty}\left\|g^{\prime}\right\|_{\infty}|\operatorname{Cov}(X, Y)| \text {. }
$$

Proof The proof follows easily from the brief outline of the main points of the proof of Theorem 4.1 in Roussas [9, p.773].

By Theorem 2.1, we establish an inequality for characteristic function (c.f.) as follows:

Theorem 2.2 If $X_{1}, \ldots, X_{m}$ are $L N Q D$ random variables with finite second moments, let $\varphi_{j}\left(t_{j}\right)$ and $\varphi\left(t_{1}, \ldots, t_{m}\right)$ be c.f.'s of $X_{j}$ and $\left(X_{1}, \ldots, X_{m}\right)$, respectively, then for all nonnegative (or nonpositive) real numbers $t_{1}, \ldots, t_{m}$,

$$
\left|\varphi\left(t_{1}, \ldots, t_{m}\right)-\prod_{j=1}^{m} \varphi_{j}\left(t_{j}\right)\right| \leq 4 \sum_{1 \leq k<l \leq m}\left|t_{k} t_{l}\right|\left|\operatorname{Cov}\left(X_{k}, X_{l}\right)\right| .
$$


Proof Write

$$
\begin{array}{r}
\left|\varphi\left(t_{1}, \ldots, t_{m}\right)-\prod_{j=1}^{m} \varphi_{j}\left(t_{j}\right)\right| \leq\left|\varphi\left(t_{1}, \ldots, t_{m}\right)-\varphi\left(t_{1}, \ldots, t_{m-1}\right) \varphi_{m}\left(t_{m}\right)\right| \\
+\left|\varphi\left(t_{1}, \ldots, t_{m-1}\right)-\prod_{j=1}^{m-1} \varphi_{j}\left(t_{j}\right)\right|=: I_{1}+I_{2} .
\end{array}
$$

Further notice that $e^{i x}=\cos (x)+i \sin (x)$. Thus,

$$
\begin{aligned}
I_{1}= & \left|\operatorname{Eexp}\left(i \sum_{j=1}^{m} t_{j} X_{j}\right)-\mathrm{E} \exp \left(i \sum_{j=1}^{m-1} t_{j} X_{j}\right) \mathrm{E} \exp \left(i t_{m} X_{m}\right)\right| \\
\leq & \left|\operatorname{Cov}\left(\cos \left(\sum_{j=1}^{m-1} t_{j} X_{j}\right), \cos \left(t_{m} X_{m}\right)\right)\right|+\left|\operatorname{Cov}\left(\sin \left(\sum_{j=1}^{m-1} t_{j} X_{j}\right), \sin \left(t_{m} X_{m}\right)\right)\right| \\
& +\left|\operatorname{Cov}\left(\sin \left(\sum_{j=1}^{m-1} t_{j} X_{j}\right), \cos \left(t_{m} X_{m}\right)\right)\right|+\left|\operatorname{Cov}\left(\cos \left(\sum_{j=1}^{m-1} t_{j} X_{j}\right), \sin \left(t_{m} X_{m}\right)\right)\right| \\
= & : I_{11}+I_{12}+I_{13}+I_{14} .
\end{aligned}
$$

By the definition of LNQD, it is easy to see that $t_{m} X_{m}$ and $\sum_{j=1}^{m-1} t_{j} X_{j}$ are NQD for $t_{1}, \ldots, t_{m}>0$. Then by Theorem 2.1 , we can obtain that

$$
I_{11} \leq\left|\operatorname{Cov}\left(\sum_{j=1}^{m-1} t_{j} X_{j}, t_{m} X_{m}\right)\right| \leq \sum_{j=1}^{m-1} t_{j} t_{m}\left|\operatorname{Cov}\left(X_{j}, X_{m}\right)\right| .
$$

Similarly as above, we have

$$
I_{1 i} \leq \sum_{j=1}^{m-1} t_{j} t_{m}\left|\operatorname{Cov}\left(X_{j}, X_{m}\right)\right|, \quad i=2,3,4 .
$$

From (2.2) to (2.4), we obtain

$$
I_{1} \leq 4 \sum_{j=1}^{m-1} t_{j} t_{m}\left|\operatorname{Cov}\left(X_{j}, X_{m}\right)\right|
$$

Therefore, in view of (2.1) and (2.5), we obtain that

$$
\left|\varphi\left(t_{1}, \ldots, t_{m}\right)-\prod_{j=1}^{m} \varphi_{j}\left(t_{j}\right)\right| \leq 4 \sum_{j=1}^{m-1} t_{j} t_{m}\left|\operatorname{Cov}\left(X_{j}, X_{m}\right)\right|+I_{2} .
$$

For $I_{2}$, using the same decomposition as in (2.1) above, we obtain

$$
\begin{aligned}
I_{2} & \leq\left|\varphi\left(t_{1}, \ldots, t_{m-1}\right)-\varphi\left(t_{1}, \ldots, t_{m-1}\right) \varphi_{m-1}\left(t_{m-1}\right)\right|+\left|\varphi\left(t_{1}, \ldots, t_{m-2}\right)-\prod_{j=1}^{m-2} \varphi_{j}\left(t_{j}\right)\right| \\
& =: I_{21}+I_{22} .
\end{aligned}
$$


Similarly to the calculation of $I_{1}$, we get

$$
I_{2} \leq 4 \sum_{j=1}^{m-2} t_{j} t_{m-1}\left|\operatorname{Cov}\left(X_{j}, X_{m-1}\right)\right|+I_{22}
$$

Thus, from (2.6) and (2.7), constantly repeating the above procedure, we get

$$
\begin{aligned}
& \left|\varphi\left(t_{1}, \ldots, t_{m}\right)-\prod_{j=1}^{m} \varphi_{j}\left(t_{j}\right)\right| \\
& \leq 4 \sum_{j=1}^{m-1} t_{j} t_{m}\left|\operatorname{Cov}\left(X_{j}, X_{m}\right)\right|+4 \sum_{j=1}^{m-2} t_{j} t_{m-1}\left|\operatorname{Cov}\left(X_{j}, X_{m-1}\right)\right| \\
& \quad+4 \sum_{j=1}^{m-3} t_{j} t_{m-2}\left|\operatorname{Cov}\left(X_{j}, X_{m-2}\right)\right|+\cdots+4\left|\operatorname{Cov}\left(X_{1}, X_{2}\right)\right| \\
& =4 \sum_{l=2}^{m} \sum_{k=1}^{l-1} t_{k} t_{l}\left|\operatorname{Cov}\left(X_{k}, X_{l}\right)\right|=4 \sum_{1 \leq k<l \leq m} t_{k} t_{l}\left|\operatorname{Cov}\left(X_{k}, X_{l}\right)\right| .
\end{aligned}
$$

Note that for $t_{1}, \ldots, t_{m}<0,-t_{m} X_{m}$ and $\sum_{j=1}^{m-1}-t_{j} X_{j}$ are NQD by the definition of LNQD. Similarly as above, we obtain that

$$
\left|\varphi\left(t_{1}, \ldots, t_{m}\right)-\prod_{j=1}^{m} \varphi_{j}\left(t_{j}\right)\right| \leq 4 \sum_{1 \leq k<l \leq m}\left|t_{k} t_{l}\right|\left|\operatorname{Cov}\left(X_{k}, X_{l}\right)\right| .
$$

This result, along with (2.8), completes the proof of the theorem.

Theorem 2.3 Let $X_{1}, \ldots, X_{n}$ be a sequence of $L N Q D$ random variables, and let $t_{1}, \ldots, t_{n}$ be all nonnegative (or nonpositive) real numbers. Then

$$
\mathrm{E}\left[\exp \left(\sum_{j=1}^{n} t_{j} X_{j}\right)\right] \leq \prod_{j=1}^{n} \mathrm{E}\left[\exp \left(t_{j} X_{j}\right)\right]
$$

Remark 2.1 Let $t_{j}=1, \forall j \geq 1$ in Theorem 2.3, we can get Lemma 3.1 of Ko et al. [5]; let $t_{j}=t>0, \forall j \geq 1$, we also get Lemma 1.4 of Wang et al. [7]. Thus, our Theorem 2.3 improves and extends Lemma 3.1 in Ko et al. [5] and Lemma 1.4 in Wang et al. [7].

Proof For $t_{1}, \ldots, t_{n}>0$, it is easy to see that $\sum_{j=1}^{i-1} t_{j} X_{j}$ and $t_{i} X_{i}$ are NQD by the definition of LNQD, which implies that $\exp \left(\sum_{j=1}^{i-1} t_{j} X_{j}\right)$ and $\exp \left(t_{i} X_{i}\right)$ are also NQD for $i=2,3, \ldots, n$ by Lemma 1.1(ii). Then by Lemma 1.1(i) and induction,

$$
\begin{aligned}
\mathrm{E}\left[\exp \left(\sum_{j=1}^{n} t_{j} X_{j}\right)\right] & \leq \mathrm{E}\left[\exp \left(\sum_{j=1}^{n-1} t_{j} X_{j}\right)\right] \mathrm{E}\left[\exp \left(t_{n} X_{n}\right)\right] \\
& =\mathrm{E}\left[\exp \left(\sum_{j=1}^{n-2} t_{j} X_{j}\right) \exp \left(t_{n-1} X_{n-1}\right)\right] \mathrm{E}\left[\exp \left(t_{n} X_{n}\right)\right]
\end{aligned}
$$




$$
\begin{aligned}
& \leq \mathrm{E}\left[\exp \left(\sum_{j=1}^{n-2} t_{j} X_{j}\right)\right] \mathrm{E}\left[\exp \left(t_{n-1} X_{n-1}\right)\right] \mathrm{E}\left[\exp \left(t_{n} X_{n}\right)\right] \\
& \leq \cdots \leq \prod_{j=1}^{n} \mathrm{E}\left[\exp \left(t_{j} X_{j}\right)\right] .
\end{aligned}
$$

For $t_{1}, \ldots, t_{n}<0$, it is easy to see that $-t_{1}, \ldots,-t_{n}>0$ and $\sum_{j=1}^{i-1}-t_{j} X_{j}$ and $-t_{i} X_{i}$ are NQD by the definition of LNQD, which implies that $\exp \left(-\sum_{j=1}^{i-1}-t_{j} X_{j}\right)$ and $\exp \left(-\left(-t_{i} X_{i}\right)\right)$ are also NQD for $i=2,3, \ldots, n$ by Lemma 1.1(ii). Similar to the proof of (2.9), we obtain

$$
\mathrm{E}\left[\exp \left(\sum_{j=1}^{n} t_{j} X_{j}\right)\right]=\mathrm{E}\left[\exp \left(-\sum_{j=1}^{n}-t_{j} X_{j}\right)\right] \leq \prod_{j=1}^{n} \mathrm{E}\left\{\exp \left[-\left(-t_{j} X_{j}\right)\right]\right\}=\prod_{j=1}^{n} \mathrm{E}\left[\exp \left(t_{j} X_{j}\right)\right]
$$

Therefore, the proof is complete by (2.9) and (2.10).

Theorem 2.4 Suppose that $\left\{X_{j}: j \geq 1\right\}$ is a LNQD random variable sequence with zero mean and $\left|X_{j}\right| \leq d_{j}$ a.s. $(j=1,2, \ldots)$. Let $t>0$ and $t \cdot \max _{1 \leq j \leq n} d_{j} \leq 1$. Then for any $\varepsilon>0$,

$$
\mathrm{P}\left(\left|\sum_{j=1}^{n} X_{j}\right| \geq \varepsilon\right) \leq 2 \exp \left\{-t \varepsilon+t^{2} \sum_{i=1}^{n} \mathrm{E} X_{j}^{2}\right\}
$$

Proof We obtain the result from the proving process of Theorem 2.3 in Wang et al. [7]

Theorem 2.5 Let $\left\{X_{j}: j \geq 1\right\}$ be a LNQD random variable sequence with zero mean and finite second moment, $\sup _{j \geq 1} \mathrm{E}\left(X_{j}^{2}\right)<\infty$. Assume that $\left\{a_{j}, j \geq 1\right\}$ is a real constant sequence satisfying $a:=\sup _{j \geq 1}\left|a_{j}\right|<\infty$. Then for any $r>1, \mathrm{E}\left|\sum_{j=1}^{n} a_{j} X_{j}\right|^{r} \leq D a^{r} n^{r / 2}$.

Proof Let $a_{i}^{+}:=\max \left\{a_{i}, 0\right\}, a_{i}^{-}:=\max \left\{-a_{i}, 0\right\}$. Notice that

$$
\begin{aligned}
& \mathrm{E}\left|\sum_{j=1}^{n} a_{j} X_{j}\right|^{r} \leq C\left\{\mathrm{E}\left|\sum_{j=1}^{n} a_{j}^{+} X_{j}\right|^{r}+\mathrm{E}\left|\sum_{j=1}^{n} a_{j}^{-} X_{j}\right|^{r}\right\}, \\
& \mathrm{E}\left|\sum_{j=1}^{n} a_{j}^{+} X_{j}\right|^{r}=a^{r} \mathrm{E}\left|\sum_{j=1}^{n} a_{j}^{+} a^{-1} X_{j}\right|^{r} .
\end{aligned}
$$

Let $Y_{j}=a_{j}^{+} a^{-1} X_{j}$. Then $\left\{Y_{n}, n \geq 1\right\}$ is still a sequence of LNQD random variables with $\mathrm{E} Y_{n}=$ 0 by Remark 1.1. Note that $0<a_{j}^{+} a^{-1} \leq 1$. By Lemma 1.2, we obtain

$$
\mathrm{E}\left|\sum_{j=1}^{n} Y_{j}\right|^{r} \leq D a^{r} n^{r / 2}, \quad \text { this implies that } \mathrm{E}\left|\sum_{j=1}^{n} a_{j}^{+} X_{j}\right|^{r} \leq D a^{r} n^{r / 2}
$$

Similarly as above, we have

$$
\mathrm{E}\left|\sum_{j=1}^{n} a_{j}^{-} X_{j}\right|^{r} \leq D a^{r} n^{r / 2}
$$

Combining (2.11)-(2.13), we get the result of the theorem. 


\section{Application}

To show the application of the inequalities in Section 2, in this section we discuss the asymptotic normality of the general linear estimator for the following regression model:

$$
Y_{n i}=g\left(x_{n i}\right)+\varepsilon_{n i}, \quad 1 \leq i \leq n,
$$

where the design points $x_{n i}, \ldots, x_{n n} \in A$, which is a compact set of $R^{d}, g$ is a bounded real valued function on $A$, and the $\left\{\varepsilon_{n i}\right\}$ are regression errors with zero mean and finite variance $\sigma^{2}$. As an estimate of $g(\cdot)$, we consider the following general linear smoother:

$$
g_{n}(x)=\sum_{i=1}^{n} w_{n i}(x) Y_{n i}
$$

where a weight function $w_{n i}(x), i=1, \ldots, n$, depends on the fixed design points $x_{n 1}, \ldots, x_{n n}$ and on the number of observations $n$.

Here, our purpose is to use the inequalities in Section 2 to establish asymptotic normality for the estimate (3.2) under LNQD condition. The results obtained generalize the results of Roussas et al. [10] and Yang [11] based on strong mixing sequence to LNQD sequence. Adopting the basic assumptions of Yang [11], we assume the following:

Assumption (A1) (i) $g: A \rightarrow R$ is a bounded function defined on the compact subset $A$ of $R^{d}$; (ii) $\left\{\xi_{t}: t=0, \pm 1, \ldots\right\}$ is a strictly stationary and LNQD time series with $\mathrm{E} \xi_{1}=0$, $\operatorname{Var}\left(\xi_{1}\right)=\sigma^{2} \in(0, \infty)$; (iii) For each $n$, the joint distribution of $\left\{\varepsilon_{n i}: 1 \leq i \leq n\right\}$ is the same as that of $\left\{\xi_{1}, \ldots, \xi_{n}\right\}$.

Denote

$$
w_{n}(x):=\max \left\{\left|w_{n i}(x)\right|: 1 \leq i \leq n\right\}, \quad \sigma_{n}^{2}(x):=\operatorname{Var}\left(g_{n}(x)\right) .
$$

Assumption (A2) (i) $\sum_{i=1}^{n}\left|w_{n i}(x)\right| \leq C$ for all $n \geq 1$; (ii) $w_{n}(x)=O\left(\sum_{i=1}^{n} w_{n i}^{2}(x)\right)$; (iii) $\sum_{i=1}^{n} w_{n i}^{2}(x)=O\left(\sigma_{n}^{2}(x)\right)$.

Assumption (A3) $\mathrm{E}\left|\xi_{1}\right|^{r}<\infty$ for $r>2$ and $u(1)=\sup _{j \geq 1} \sum_{|i-j| \geq 1}\left|\operatorname{Cov}\left(\xi_{i}, \xi_{j}\right)\right|<\infty$.

Assumption (A4) There exist positive integers $p:=p(n)$ and $q:=q(n)$ such that $p+q \leq n$ for sufficiently large $n$ and as $n \rightarrow \infty$,
(i) $q p^{-1} \rightarrow 0$;
(ii) $n q p^{-1} w_{n} \rightarrow 0$;
(iii) $p w_{n} \rightarrow 0$
(iv) $n p^{\frac{r}{2}-1} w_{n}^{\frac{r}{2}} \rightarrow 0$.

Here, we will prove the following result.

Theorem 3.1 Let Assumptions (A1) (A4) be satisfied. Then

$$
\sigma_{n}^{-1}(x)\left\{g_{n}(x)-\mathrm{E} g_{n}(x)\right\} \stackrel{d}{\rightarrow} N(0,1)
$$

Proof We first give some denotations. For convenience of writing, omit everywhere the argument $x$ and set $S_{n}=\sigma_{n}^{-1}\left(g_{n}-\mathrm{E} g_{n}\right), Z_{n i}=\sigma_{n}^{-1} w_{n i} \varepsilon_{n i}$ for $i=1, \ldots, n$, so that $S_{n}=\sum_{i=1}^{n} Z_{n i}$. 
Let $k=[n /(p+q)]$. Then $S_{n}$ may be split as $S_{n}=S_{n}^{\prime}+S_{n}^{\prime \prime}+S_{n}^{\prime \prime \prime}$, where

$$
\begin{aligned}
& S_{n}^{\prime}=\sum_{m=1}^{k} y_{n m}, \quad S_{n}^{\prime \prime}=\sum_{m=1}^{k} y_{n m}^{\prime}, \quad S_{n}^{\prime \prime \prime}=y_{n k+1}^{\prime}, \\
& y_{n m}=\sum_{i=k_{m}}^{k_{m}+p-1} Z_{n i}, \quad y_{n m}^{\prime}=\sum_{i=l_{m}}^{l_{m}+q-1} Z_{n i}, \quad y_{n k+1}^{\prime}=\sum_{i=k(p+q)+1}^{n} Z_{n i}, \\
& k_{m}=(m-1)(p+q)+1, \quad l_{m}=(m-1)(p+q)+p+1, \quad m=1, \ldots, k .
\end{aligned}
$$

Thus, to prove the theorem, it suffices to show that

$$
\begin{aligned}
& \mathrm{E}\left(S_{n}^{\prime \prime}\right)^{2} \rightarrow 0, \quad \mathrm{E}\left(S_{n}^{\prime \prime \prime}\right)^{2} \rightarrow 0, \\
& S_{n}^{\prime} \stackrel{d}{\rightarrow} N(0,1) .
\end{aligned}
$$

By Theorem 2.5, Assumptions (A2)(ii) (iii) and (A4)(i) (iii), we have

$$
\begin{aligned}
& \mathrm{E}\left(S_{n}^{\prime \prime}\right)^{2}=\mathrm{E}\left(\sum_{m=1}^{k} \sum_{i=l_{m}}^{l_{m}+q-1} \sigma^{-1} w_{n i} \xi_{i}\right)^{2} \leq D k q \sigma^{-2} w_{n}^{2} \leq C\left(1+q p^{-1}\right)^{-1} n q p^{-1} w_{n} \rightarrow 0, \\
& \mathrm{E}\left(S_{n}^{\prime \prime \prime}\right)^{2}=\mathrm{E}\left(\sum_{i=k(p+q)+1}^{n} \sigma^{-1} w_{n i} \xi_{i}\right)^{2} \leq D(n-k(p+q)) \sigma^{-2} w_{n}^{2} \leq C\left(1+q p^{-1}\right) p w_{n} \rightarrow 0 .
\end{aligned}
$$

Thus (3.4) holds.

We now proceed with the proof of (3.5). Let $\Gamma_{n}=\sum_{1 \leq i<j \leq k} \operatorname{Cov}\left(y_{n i}, y_{n j}\right)$ and $s_{n}^{2}=$ $\sum_{m=1}^{k} \operatorname{Var}\left(y_{n m}\right)$, then $s_{n}^{2}=\mathrm{E}\left(S_{1 n}^{\prime}\right)^{2}-2 \Gamma_{n}$. Apply relation (3.4) to obtain $\mathrm{E}\left(S_{n}^{\prime}\right)^{2} \rightarrow 1$. This would also imply that $s_{n}^{2} \rightarrow 1$, provided we show that $\Gamma_{n} \rightarrow 0$.

Indeed, by Assumption (A3) and $u(1)<\infty$, we obtain $u(q) \rightarrow 0$. Then by stationarity and Assumption (A2), it can be shown that

$$
\begin{aligned}
\left|\Gamma_{n}\right| & \leq \sum_{1 \leq i<j \leq k} \sum_{\mu=k_{i}}^{k_{i}+p-1} \sum_{\nu=k_{j}}^{k_{j}+p-1}\left|\operatorname{Cov}\left(Z_{n \mu}, Z_{n v}\right)\right| \\
& \leq \sum_{1 \leq i<j \leq k} \sum_{\mu=k_{i}}^{k_{i}+p-1} \sum_{\nu=k_{j}}^{k_{j}+p-1} \sigma_{n}^{-2}\left|w_{n \mu} w_{n v}\right| \cdot\left|\operatorname{Cov}\left(\xi_{\mu}, \xi_{v}\right)\right| \\
& \leq C \sigma_{n}^{-2} w_{n} \sum_{i=1}^{k-1} \sum_{\mu=k_{i}}^{k_{i}+p-1}\left|w_{n \nu}\right| \cdot \sup _{j \geq 1} \sum_{t:|t-j| \geq q}\left|\operatorname{Cov}\left(\xi_{j}, \xi_{t}\right)\right| \leq \operatorname{Cu}(q) \rightarrow 0 .
\end{aligned}
$$

Next, in order to establish asymptotic normality, we assume that $\left\{\eta_{n m}: m=1, \ldots, k\right\}$ are independent random variables, and the distribution of $\eta_{n m}$ is the same as that $y_{n m}$ for $m=1, \ldots, k$. Then $\mathrm{E} \eta_{n m}=0$ and $\operatorname{Var}\left(\eta_{n m}\right)=\operatorname{Var}\left(y_{n m}\right)$. Let $T_{n m}=\eta_{n m} / s_{n}, m=1, \ldots, k$, then $\left\{T_{n m}, m=1, \ldots, k\right\}$ are independent random variables with $E T_{n m}=0$ and $\operatorname{Var}\left(T_{n m}\right)=1$. Let 
$\varphi_{X}(t)$ be the characteristic function of $X$, then

$$
\begin{aligned}
& \left|\phi_{\sum_{m=1}^{k} y_{n m}}(t)-e^{-\frac{t^{2}}{2}}\right| \\
& \leq\left|\operatorname{Eexp}\left(i t \sum_{m=1}^{k} y_{n m}\right)-\prod_{m=1}^{k} \mathrm{E} \exp \left(i t y_{n m}\right)\right|+\left|\prod_{m=1}^{k} \mathrm{E} \exp \left(i t y_{n m}\right)-e^{-\frac{t^{2}}{2}}\right| \\
& \leq\left|\operatorname{Eexp}\left(i t \sum_{m=1}^{k} y_{n m}\right)-\prod_{m=1}^{k} \mathrm{E} \exp \left(i t y_{n m}\right)\right|+\left|\prod_{m=1}^{k} \mathrm{E} \exp \left(i t \eta_{n m}\right)-e^{-\frac{t^{2}}{2}}\right|=: I_{3}+I_{4} .
\end{aligned}
$$

By Theorem 2.2, relation (3.6) and Assumption (A2), we obtain that

$$
I_{3} \leq 4 t^{2} \sum_{1 \leq i<j \leq k} \sum_{\mu=k_{i}}^{k_{i}+p-1} \sum_{\nu=k_{j}}^{k_{j}+p-1}\left|\operatorname{Cov}\left(Z_{n \mu}, Z_{n v}\right)\right| \leq C u(q) \rightarrow 0 .
$$

Thus, it suffices to show that $\eta_{n m} \stackrel{d}{\rightarrow} N(0,1)$ which, on account of $s_{n}^{2} \rightarrow 1$, will follow from the convergence $\sum_{m=1}^{k} T_{n m} \stackrel{d}{\rightarrow} N(0,1)$. By the Lyapunov condition, it suffices to show that for some $r>2$,

$$
\frac{1}{s_{n}^{r}} \sum_{m=1}^{k} \mathrm{E}\left|\eta_{n m}\right|^{r} \rightarrow 0
$$

Using Theorem 2.5 and Assumptions (A2) and (A4)(iv), we have

$$
\begin{aligned}
\sum_{m=1}^{k} \mathrm{E}\left|\eta_{n m}\right|^{r} & =\sum_{m=1}^{k} \mathrm{E}\left|y_{n m}\right|^{r}=\sum_{m=1}^{k} \mathrm{E}\left|\sum_{i=k_{m}}^{k_{m}+p-1} \sigma_{n}^{-1} w_{n i} \xi_{i}\right|^{r} \\
& \leq D k \sigma_{n}^{r} w_{n}^{r} p^{\frac{r}{2}} \leq C n p^{\frac{r}{2}-1} w_{n}^{\frac{r}{2}} \rightarrow 0 .
\end{aligned}
$$

So, (3.9) holds. Thus, the proof is complete.

\section{Competing interests}

The authors declare that they have no competing interests.

\section{Authors' contributions}

The three authors contributed equally to this work. All authors read and approved the final manuscript.

\section{Author details}

${ }^{1}$ Department of Mathematics, Shangrao Normal University, Shangrao, 334001, China. ${ }^{2}$ Department of Mathematics, Guangdong Ocean University, Zhanjiang, Guangdong 524088, China.

\section{Acknowledgements}

This research is supported by the National Natural Science Foundation of China (11061029) and the Science Foundation of Jiangxi Education Department (GJJ12604).

Received: 3 February 2012 Accepted: 18 September 2012 Published: 2 October 2012

\section{References}

1. Lehmann, EL: Some concepts of dependence. Ann. Math. Stat. 37, 1137-1153 (1966)

2. Newman, CM: Asymptotic independence and limit theorems for positively and negatively dependent random variables. In: Tong, YL (ed.) Statistics and Probability, vol. 5, pp. 127-140. Inst. Math. Statist., Hayward (1984)

3. Joag-Dev, K, Proschan, F: Negative association of random variables with applications. Ann. Stat. 11(1), $286-295$ (1983) 
4. Zhang, LX: A functional central limit theorem for asymptotically negatively dependent random fields. Acta Math. Hung. 86, 237-259 (2000)

5. Ko, MH, Choi, YK, Choi, YS: Exponential probability inequality for linearly negative quadrant dependent random variables. Commun. Korean Math. Soc. 22, 137-143 (2007)

6. Ko, MH, Ryu, DH, Kim, TS: Limiting behaviors of weighted sums for linearly negative quadrant dependent random variables. Taiwan. J. Math. 11(2), 511-522 (2007)

7. Wang, XJ, Hu, SH, Yang, WZ, Li, XQ: Exponential inequalities and complete convergence for a LNQD sequence. J. Korean Stat. Soc. 39, 555-564 (2010)

8. Wu, YF, Guan, M: Mean convergence theorems and weak laws of large numbers for weighted sums of dependent random variables. J. Math. Anal. Appl. 377, 613-623 (2011)

9. Roussas, GG: Positive and negative dependence with some statistical applications. In: Ghosh, S, Puri, ML (eds.) Asymptotics, Nonparametrics, and Time Series, pp. 757-787. CRC Press, Boca Raton (1999)

10. Roussas, GG, Tran, LT, loannides, DA: Fixed design regression for time series: asymptiotic normality. J. Multivar. Anal. 40, 162-291 (1992)

11. Yang, SC: Maximal moment inequality for partial sum of strong mixing sequences and application. Acta Math. Sin. Engl. Ser. 23(6), 1013-1024 (2007)

doi:10.1186/1029-242X-2012-216

Cite this article as: Li et al.: Some inequalities for a LNQD sequence with applications. Journal of Inequalities and Applications 2012 2012:216.

\section{Submit your manuscript to a SpringerOpen ${ }^{\ominus}$ journal and benefit from:}

- Convenient online submission

- Rigorous peer review

- Immediate publication on acceptance

- Open access: articles freely available online

- High visibility within the field

- Retaining the copyright to your article 